\title{
Secondary Data Analysis of Tuberculosis Deaths in Bulawayo Province, Zimbabwe, 2016-2019
}

\author{
Tshebukani Mzingaye Moyo1, Edwin Sibanda², Notion Tafara Gombe ${ }^{1,3}$, Tsitsi Patience Juru1, \\ Emmanuel Govha1, Maurice Omondi' ${ }^{4}$, Addmore Chadambuka1 ${ }^{*}$, Mufuta Tshimanga ${ }^{1}$
}

${ }^{1}$ Department of Primary Health Care Sciences, Global and Public Health, University of Zimbabwe, Harare, Zimbabwe

${ }^{2}$ Bulawayo City Council Department of Health, Bulawayo, Zimbabwe

${ }^{3}$ African Field Epidemiology Network, Harare, Zimbabwe

${ }^{4}$ African Field Epidemiology Network, Nairobi, Kenya

Email: *achadambuka1@yahoo.co.uk

How to cite this paper: Moyo, T.M., Sibanda, E., Gombe, N.T., Juru, T.P., Govha, E., Omondi, M., Chadambuka, A. and Tshimanga, M. (2022) Secondary Data Analysis of Tuberculosis Deaths in Bulawayo Province, Zimbabwe, 2016-2019. Open Journal of Epidemiology, 12, 57-67.

https://doi.org/10.4236/ojepi.2022.121005

Received: December 1, 2021

Accepted: February 5, 2022

Published: February 8, 2022

Copyright $\odot 2022$ by author(s) and Scientific Research Publishing Inc. This work is licensed under the Creative Commons Attribution International License (CC BY 4.0).

http://creativecommons.org/licenses/by/4.0/

\section{(c) (i) Open Access}

\begin{abstract}
Background: Tuberculosis is a leading cause of death globally, and the third leading cause of death in Zimbabwe. Death from any cause following a diagnosis of tuberculosis is classified as a tuberculosis death. Bulawayo Province reported high tuberculosis death rates from $15.3 \%$ in 2016 to $14.2 \%$ in 2019 against a threshold of $5 \%$. We analyzed tuberculosis deaths for Bulawayo Province to characterize patients dying and to make recommendations for improving treatment outcomes for susceptible tuberculosis cases. Methods: A descriptive cross-sectional study was conducted. We analyzed all $(\mathrm{N}=469)$ records of tuberculosis deaths from 19/19 Bulawayo tuberculosis diagnosing centers from 01 January 2016 to 31 December 2019. Microsoft ${ }^{\circledR}$ Excel 2007 was used to generate graphs and Stata ${ }^{\circledR}$ version 17 was used to conduct chi-square tests for trends. Results: Males accounted for 278/469 (59.3\%) of the deaths. The median age of death was 40 years $\left(\mathrm{Q}_{1}=33: \mathrm{Q}_{3}=51\right)$. The proportion of TB deaths increased from 63/114 (55\%) in 2016 to $57 / 90$ (63\%) in 2019 for males $(\mathrm{p}<0.01)$. The majority of deaths $278 / 469(59.3 \%)$ occurred in the intensive phase of treatment and anemia was a co-morbid condition in only $44 / 469$ (9.4\%) of the tuberculosis deaths. Testing for anemia was not routinely done. Conclusion: High death rates particularly in the intensive phase, could be attributed to sub-optimal clinical care. Tuberculosis programs should work towards adopting differentiated care models for tuberculosis patients and developing algorithms for patients at high risk of death.
\end{abstract}

\section{Keywords}

Tuberculosis Death, Secondary Data Analysis, Bulawayo Province, Zimbabwe 


\section{Introduction}

Death from any cause in a person who had a diagnosis of tuberculosis (TB) is classified as a tuberculosis death [1]. The disease is caused by the bacilli Mycobacterium tuberculosis. Tuberculosis is a preventable communicable disease and ranks amongst the top ten causes of death globally [2]. According to the World Health Organization (WHO), the world had an estimated 1,418,000 deaths in 2019 , with $95 \%$ of these deaths being reported by low to middle-income countries. About $85 \%$ of people who develop TB can be successfully treated with a six months drug regimen, and since the year 2000, the use of anti-tuberculosis medicines has managed to avert more than 60 million deaths [2]. Death during TB treatment is not merely a function of infection with Mycobacterium tuberculosis but there are host, disease, and health system factors that contribute to the negative outcome [1]. The majority of the TB deaths are ascribed to non-tuberculosis-related causes. Therefore, treating TB and the underlying co-morbidities is essential to ensure positive outcomes for the enrolled cases [3].

Tuberculosis is the third leading cause of death in Zimbabwe, and the country reported 4600 deaths in 2019 [2] [4]. Zimbabwe has suffered multiple economic crises in the last decade, and the economy remains fragile and characterized by retrenchments and widening poverty [5]. More than a decade of economic meltdown and rising costs have had a telling effect on a once vibrant health system [6]. Despite these challenges, the country is working towards reducing the $\mathrm{TB}$ death rate to not more than five percent for all registered cases [2] [4]. Bulawayo Province reported high tuberculosis death rates from 276/1902 (15.3\%) in 2016 to $225 / 1637(14.2 \%)$ in 2019 . This is despite interventions like TB case management training, directly observed treatment (DOT), TB and Human Immunodeficiency Virus (HIV) collaboration, and TB death audits.

The goal of reducing TB deaths can be achieved if TB deaths are considered predictable and preventable. Programs should examine and address some key gaps in the understanding of the distribution and determinants of TB deaths [1]. In Zimbabwe, all TB deaths should be audited within seven days by the health workers at the facility the deceased patient was receiving treatment. The audit forms are consolidated at the provincial level, and quarterly reports are submitted to the national level [7]. We analyzed TB deaths for Bulawayo Province to characterize patients dying, determine the reasons for the high death rates, and make recommendations to improve treatment outcomes for susceptible TB patients.

\section{Methods}

\subsection{Study Design}

We conducted a descriptive cross-sectional study.

\subsection{Study Setting}

Bulawayo Metropolitan Province is one of the ten provinces of Zimbabwe and 
has a population of 707,470 according to the 2020 Zimbabwe National Statistics Agency (ZIMSTAT) population estimates [8]. It has three administrative districts namely Emakhandeni, Nkulumane, and Northern Suburbs. The province has 19 health facilities that offer tuberculosis prevention, treatment, and care services. Bulawayo Province borders Matabeleland's North and South provinces and has proximity to South Africa, and Botswana (Figure 1). Due to limited opportunities for gainful employment within the province, residents seek opportunities in these neighboring countries.

\subsection{Study Population}

We used data collected from TB patients who died in Bulawayo Province from 01 January 2016 to 31 December 2019. Five key informants namely the deputy chief nursing officer, a clinical medical officer, a laboratory technician, a pharmacy technician, and a TB nurse were purposively selected.

\subsection{Data Source}

Health workers collect data using the TB death audit form for all TB deaths. The primary purpose for recording the data is for evaluating the TB program's performance. Variables captured include age, sex, HIV status, type of tuberculosis, other existing co-morbid conditions, and phase of treatment when the patient died. Health care workers capture the variables on a hard copy death audit tool. Copies are submitted to the health information section for consolidation and onwards transmission to the Ministry of health and child care's head office in Harare. An individual TB death record was a study unit.

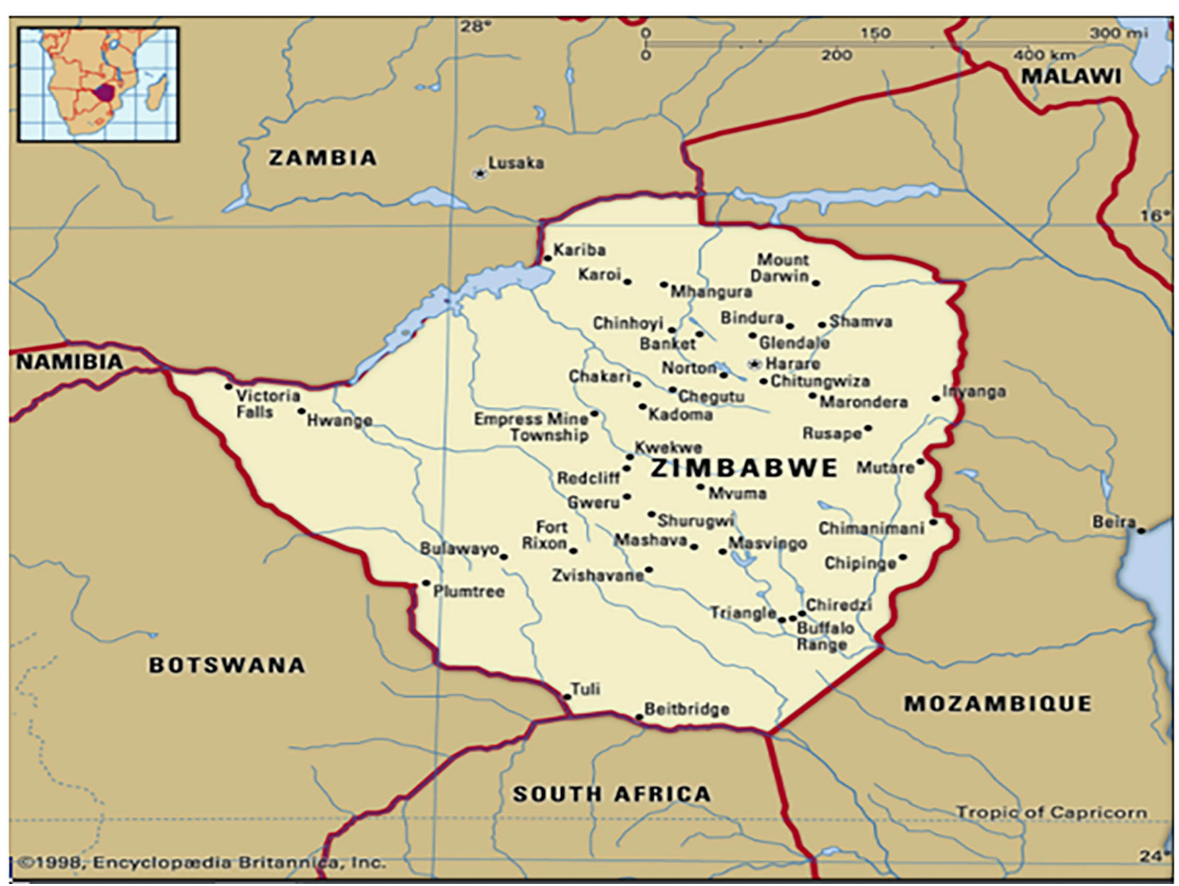

Figure 1. Map of Zimbabwe showing the proximity of Bulawayo province to Botswana and South Africa. Source: Encyclopaedia Britannica, Accessed 15 June 2021. 


\subsection{Data Collection}

The TB death audit forms were reviewed and data were captured on created line lists. We entered all the 469 susceptible TB death records for the period 01 January 2016 to 31 December 2019. Only 4/471 (0.8\%) patients who died had drug-resistant tuberculosis. As such, records of patients with drug-resistant tuberculosis were excluded. A key-informant guide was used to conduct interviews to determine the reasons for the high death rates in the province. As part of the key informant interviews, a checklist was used to collect information on the availability of laboratory personnel and requisite equipment and consumables for key laboratory tests. Physical inspection to assess for the availability of full blood count (FBC), chemistry analyzer, and reagents for $\mathrm{FBC}$, urea and electrolytes (UandEs), liver function tests (LFTs), and blood grouping was done. Stock cards for these reagents were also inspected to assess stock monitoring and control by the laboratory staff.

\subsection{Data Analysis}

We used Microsoft ${ }^{\circledR}$ Office Excel 2007 to generate graphs. The Stata ${ }^{\circledR} 17$ statistical software was used to perform chi-square tests for trends of proportions at a significance level of $\mathrm{p}<0.05$. Data quality was assessed by checking for completeness of the audit forms. For records with missing information for certain variables, we excluded that record in the analysis of the missing variable. Key themes and powerful quotes on the cause of the high TB death rates in the province were identified and reported. Data on laboratory services collected using the checklist were analyzed to determine which services were not available to the TB patients.

\subsection{Permission and Ethical Considerations}

Permission to carry out the study was sought and obtained from the Director of Health Services Bulawayo City Council, and the Health Studies Office. Confidentiality was maintained as the names of the key informants interviewed were not recorded on the key informant guide.

\section{Results}

\subsection{Data Analysis}

\subsubsection{Data Quality}

A total of 469 tuberculosis death records were line-listed and analyzed. The reviewed death audit forms had most variables filled in $460 / 469$ (98\%) and the remaining records $9 / 469(2 \%)$ had missing data on co-morbid conditions.

\subsubsection{Trends and Distribution of TB Deaths}

Demographic characteristics of TB patients who died

Males accounted for 278/469 (59.3\%) of the deaths. The median age of death was 40 years $\left(\mathrm{Q}_{1}=33, \mathrm{Q}_{3}=51\right)$. The most affected age groups were the $25-34$ 
years, 35 - 44 years, and 45 - 54 years with 118/469 (25.2\%), 143/469 (30.5\%), and 90/469 (19.2\%) tuberculosis deaths respectively (Table 1).

Tuberculosis deaths among genders

Tuberculosis deaths declined from $51 / 114$ (45\%) in 2016 to $33 / 90$ (37\%) in 2019 for females and increased from 63/114 (55\%) in 2016 to 57/90 (63\%) in 2019 for males. There was a significant upward linear trend for the proportion of TB deaths occurring amongst men $(\mathrm{p}<0.01)$ (Figure 2$)$.

Co-morbid conditions

Table 1. Demographic characteristics of the TB patients who demised, Bulawayo province, 2016-2019.

\begin{tabular}{|c|c|c|}
\hline Variable & Category & Frequency/n $=469(\%)$ \\
\hline \multirow{2}{*}{ Gender } & Female & $191(40.7)$ \\
\hline & Male & $278(59.3)$ \\
\hline \multirow{2}{*}{ Treatment category } & New & $399(85.1)$ \\
\hline & Retreatment & $70(14.9)$ \\
\hline \multirow{9}{*}{ Age group } & Under five years & $2(0.4)$ \\
\hline & $5-14$ years & $3(0.6)$ \\
\hline & 15 - 20 years & $11(2.3)$ \\
\hline & $21-24$ years & $13(2.8)$ \\
\hline & 25 - 34 years & $118(25.2)$ \\
\hline & $35-44$ years & $143(30.5)$ \\
\hline & $45-54$ years & $90(19.2)$ \\
\hline & $55-64$ years & $53(11.3)$ \\
\hline & 65 years and above & $36(7.7)$ \\
\hline
\end{tabular}

Median age in years $40\left(\mathrm{Q}_{1}=33 ; \mathrm{Q}_{3}=51\right)$

$\mathrm{n}$ frequency of the tuberculosis deaths, \% per cent, $\mathrm{Q}_{1}$ first quartile, $\mathrm{Q}_{3}$ third quartile.

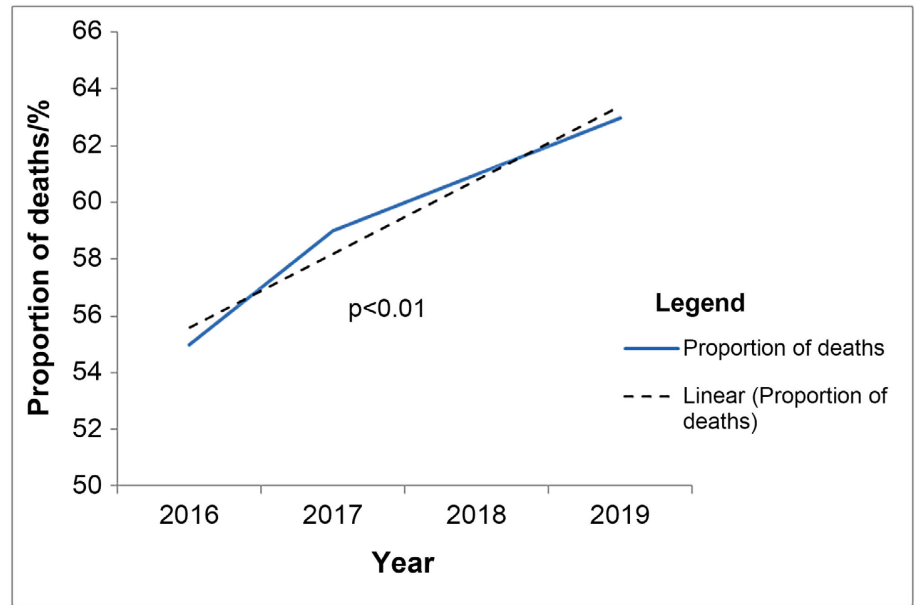

Figure 2. Trend of tuberculosis deaths in males, Bulawayo province, Zimbabwe, 2016-2019. 
The commonest co-morbid condition was being HIV co-infected 416/469 (88.7\%). Less than a tenth $44 / 469(9.4 \%)$ of the tuberculosis deaths had anemia as a co-morbid condition (Table 2). The proportion of TB/HIV co-infected patients who died declined from 101/114 (89\%) in 2016 to $71 / 90$ (79\%) in 2019, and the downward linear trend was statistically significant $(\mathrm{p}<0.01)$ (Figure 3$)$.

Distribution of TB deaths by type of TB, and treatment phase

Approximately two-thirds of the tuberculosis deaths 306/469 (65.2\%) occurred among patients with pulmonary tuberculosis. The majority of the reported deaths 278/469 (59.3\%) occurred during the intensive phase of treatment (Table 2). There was a significant upward linear trend in the proportion of TB deaths occurring in the intensive phase of treatment from 54/114 (47\%) in 2016 to $52 / 90(58 \%)$ in $2019(\mathrm{p}<0.01)$ (Figure 4$)$.

Table 2. Common co-morbid conditions, type of TB, and treatment phase, Bulawayo province, 2016-2019.

\begin{tabular}{ccc}
\hline Variable & Category & Frequency/n $=\mathbf{4 6 9}(\%)$ \\
\hline & HIV & $416(88.7)$ \\
Co-morbid & Anaemia & $44(9.4)$ \\
conditions & Conal failure & $18(3.8)$ \\
& Meningitis & $18(3.8)$ \\
& Malignancies & $17(3.6)$ \\
& Hypertension & $14(3.0)$ \\
\hline Type of TB & Diabetes Mellitus & $12(2.6)$ \\
& Pulmonary TB & $7(1.5)$ \\
\hline TB treatment phase & Extra-pulmonary TB & $306(65.2)$ \\
at time of death & Intensive phase & $163(34.8)$ \\
\hline
\end{tabular}

$\mathrm{n}$ frequency of the tuberculosis deaths, \% per cent, HIV Human Immunodeficiency Virus, TB Tuberculosis.

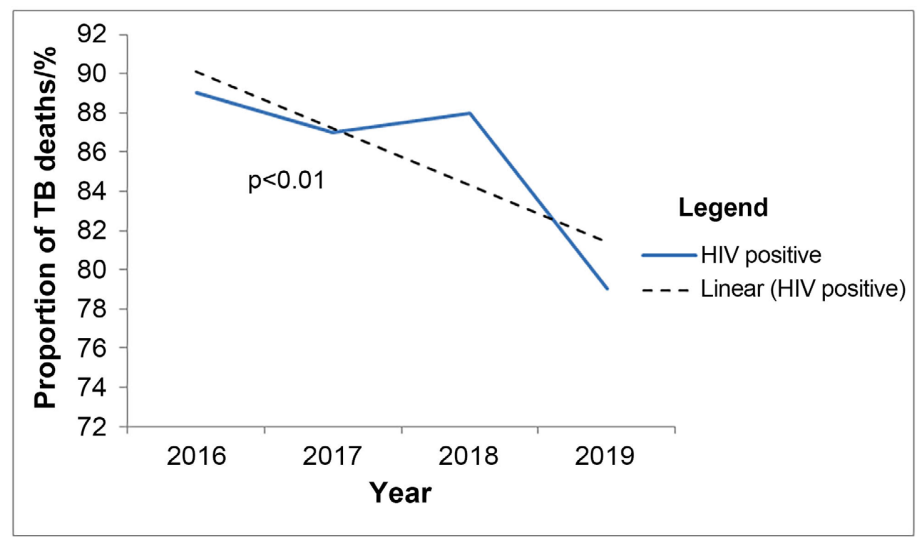

Figure 3. Trend of tuberculosis deaths among HIV positive patients, Bulawayo province, Zimbabwe, 2016-2019. 


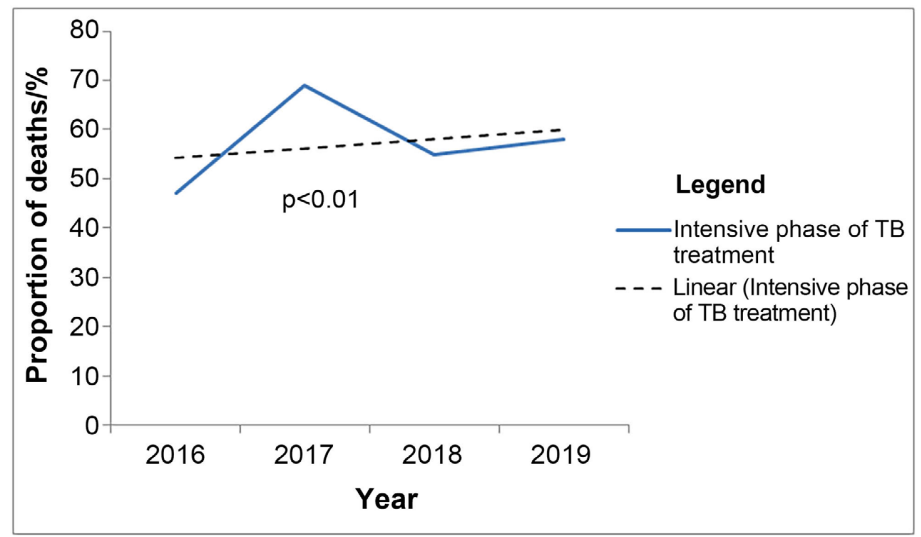

Figure 4. Trend of tuberculosis deaths in the intensive phase of tuberculosis treatment, Bulawayo province, Zimbabwe, 2016-2019.

\subsubsection{Key Informant Interviews}

Reasons for the high tuberculosis deaths

Four key informants reported that the high TB death rates could be attributed to the delayed health-seeking behavior by community members particularly men. A clinician noted that "most men present when their health has remarkably deteriorated". Three key informants attributed the high TB death rate to patients defaulting treatment and moving to neighboring countries. One of the key informants noted that "After a few weeks of treatment the TB patients will start to feel better. Due to the difficult economic situation in Zimbabwe, some of the patients leave the country to seek employment opportunities in Botswana or South Africa. Only to return when their condition has deteriorated". Two key informants identified poor access to diagnostic services for other co-morbid conditions like anemia, and renal failure as the other reason for the high tuberculosis deaths. A clinician noted that "Sometimes we end up referring the patients to the private sector due to the stock-outs of reagents in the public sector".

Laboratory services

One of the laboratory personnel noted that Bulawayo City Council's laboratory at Thorngrove hospital had the requisite manpower and equipment for tests like FBC, UandEs, LFTs, and Blood grouping. The physical inspection and review of stock cards revealed that reagents for UandEs, LFTs, and blood grouping were out-of-stock and the laboratory was under-stocked for FBC reagents.

\section{Discussion}

We conducted a secondary data set analysis for susceptible TB deaths in Bulawayo Metropolitan Province, which is one of the provinces of Zimbabwe reporting the highest TB death rates. In this study, which was conducted in a low-income country, the major findings were that: there was a significant increase in the proportion of tuberculosis deaths among males, and for patients in the intensive phase of treatment, the majority of the deaths occurred among males, HIV co-infected patients, and among patients in the intensive phase of 
treatment, and the availability of tests for co-morbid conditions like anemia, and renal failure was affected by the shortages of reagents.

Males accounted for the majority of tuberculosis deaths, and there was an increase in the proportion of reported TB deaths among men. Cultural and religious norms primarily place the role of fending for the family on men. The tuberculosis treatment duration is generally long and this can become a barrier in taking care of their families for some men who are the principal breadwinners. Due to the economic challenges in Zimbabwe, and the proximity of Bulawayo Province to neighboring countries some patients migrate to seek employment opportunities in these countries before they complete treatment. Migrants from Zimbabwe to neighboring countries are supporting the livelihoods of relatives left at home through remittances. More migrant men have been shown to remit to their families back in Zimbabwe compared to their women counterparts [9]. This could suggest that men have more pressure to get back to their vocations to fend for their families. Our findings were consistent with Washington et al., in India who found that being male was one of the higher risk categories for death [10].

Despite the significant decline in the proportion of TB deaths among HIV co-infected patients, a majority of the deaths occurred among HIV co-infected patients. The classification of TB as an HIV clinical stage three disease, and the implementation of the HIV differentiated care models has improved HIV treatment access for TB/HIV co-infected patients. The HIV programmes across the globe are implementing differentiated care models following the realization that patients are different and require individualized care [10]. These models have improved the quality of care for HIV-positive recipients of care. Implementation of these models has ensured that health workers spend more time attending to the new patients, as well as to clinically unstable patients. Tuberculosis patients are different, and may, therefore, require individualized treatment approaches. Failure to deliberately individualize the care of TB patients particularly in resource-limited countries, which are also TB high-burden countries may lead to the poor quality of care for TB patients. Resource-constrained countries are also experiencing human resources for health challenges which have been exacerbated by the Coronavirus disease (COVID-19) pandemic [11]. The implementation of the differentiated care models for TB patients will result in the efficient utilization of human resources.

The majority of the TB deaths occurred during the intensive phase of treatment, and there was an increase in the proportion of tuberculosis deaths occurring in the intensive phase of treatment from 2016-2019. This could be attributed to delayed health-seeking behavior, and patients defaulting treatment. Several studies have demonstrated that some patients present late to health facilities, and only present when their illnesses have advanced [12] [13]. The increase in the proportion of TB deaths in the intensive phase of TB treatment poses a serious challenge to the global end TB strategy targets [2]. Identification of the 
modifiable factors associated with death among TB patients in the intensive phase is critical and can provide a solid basis for targeted interventions. Our findings were consistent with Alobu et al., 2014 in Nigeria who found that the majority of TB deaths occurred in the intensive phase of treatment [14].

The prevalence of anemia as a co-morbid condition among the TB patients who died was very low. The low prevalence of anemia as a co-morbid condition in our study could be attributed to limited testing capacity for anemia as the laboratory was under-stocked for FBC reagents. The low testing capacity for anemia in the public sector could lead to anemia being missed. Anemia is known to exist as a co-morbid condition in TB patients, and the prevalence ranges between 30\% - 94\% [15]. In Zimbabwe, TB patients are exempted from paying for laboratory investigations including tests for anemia [7]. However, despite this progressive policy being in place, implementation is affected by the erratic supply of reagents. Due to the public health care under-funding, TB patients are sometimes requested to get some laboratory investigations done by private laboratories. The majority of the clients referred to the private laboratories may not afford these services. This is because tuberculosis is, by all means, a poverty disease, mainly affecting the most vulnerable populations [2] [16].

\section{Study Limitations}

We could not assess the effect of body mass index, and delayed presentation on tuberculosis deaths as the body mass index, and the date of symptom onset was not being captured on the audit tool. These are important variables that have been shown from other studies to affect the treatment outcomes for tuberculosis patients. Patients at risk of clinical deterioration after starting tuberculosis treatment are identifiable using simple measurements including the body mass index [17]. Audio recordings were not done for the key informant interviews. This was a limitation as some of the information from the key informants could have been missed during notes taking. To minimize this effect, soon after each key informant interview we reviewed the notes and expanded on the note-taking shorthand, and added details whilst we still remembered.

\section{Conclusions}

Tuberculosis deaths disproportionately affect the poor, and high death rates in these groups are also attributed to the lack of access to quality care [1]. TB patients are not homogenous and as such, the care of TB patients should not be overly standardized.

There are several programmatic implications from this study. The TB control program should consider revising the TB death audit form to capture variables like the date of symptom onset and the body mass index. Based on the study findings, TB programs should consider working towards adopting differentiated care models (patient-centered treatment models) for TB patients, taking lessons from the anti-retroviral therapy program. Countries like Zimbabwe should also 
consider developing algorithms to identify TB patients at high risk of death, and further analytical or quasi-experimental studies particularly for patients in the intensive phase are proposed. The quality of care for tuberculosis patients should also be improved through the uninterrupted availability of laboratory services.

\section{Acknowledgements}

We would like to acknowledge the staff and management of the Bulawayo City health department, and the staff of the University of Zimbabwe, the Faculty of Medicine, and Health Sciences, and the Health studies office for all the assistance rendered.

\section{Conflicts of Interest}

The authors declare no conflicts of interest regarding the publication of this paper.

\section{References}

[1] Bhargava, A. sand Bhargava, M. (2020) Tuberculosis Deaths Are Predictable and Preventable: Comprehensive Assessment and Clinical Care Is the Key. Journal of Clinical Tuberculosis and other Mycobacterial Diseases, 19, Article ID: 100155. https://doi.org/10.1016/j.jctube.2020.100155

[2] World Health Organization (2020) 2020 Global Tuberculosis Report. https://www.who.int/publications-redirect/9789240013131

[3] Lin, C.-H., Lin, C.-J., Kuo, Y.-W., Wang, J.-Y, Hsu, C.-L., Chen, J.-M., et al. (2014) Tuberculosis Mortality: Patient Characteristics and Causes. Biomedical Central Infectious Diseases, 14, Article No. 5. https://doi.org/10.1186/1471-2334-14-5

[4] Ministry of Health and Child Care (2016) National Tuberculosis Program Strategic Plan 2017-2020. https://depts.washington.edu/edgh/zw/hit/web/project-resources/TB-NSP.pdf

[5] Ministry of Health and Child Care (2020) National Tuberculosis and Leprosy Programme Strategic Plan 2021-2025.

[6] United States Agency International Development (2015) Zimbabwe Country Development Cooperation Strategy 2016-2021. https://www.usaid.gov/sites/default/files/documents/1860/Zimbabwe CDCS 20162021.pdf

[7] Ministry of Health and Child Care (2016) Zimbabwe National Tuberculosis and Leprosy Management Guidelines.

https://scribd.com/document/355736735/Zimbabwe-National-Tuberculosis-and-Le prosy-Management-Guidelinesfinal

[8] Zimbabwe National Statistics Agency (2015) Population Projections Thematic Report. Zimbabwe National Statistics Agency, Harare.

https://zimstat.co.zw/wp-content/uploads/publications/Population/population/pop ulation-projection.pdf

[9] Makina, D. (2013) Migration and Characteristics of Remittance Senders in South Africa. International Migration, 51, e148-e158.

https://doi.org/10.1111/j.1468-2435.2012.00746.x

[10] Washington, R., Potty, R.S., Rajesham, A., Seenappa, T., Singarajipura, A., Swa- 
mickan, R., et al. (2020) Is a Differentiated Care Model Needed for Patients with TB? A Cohort Analysis of Risk Factors Contributing to Unfavourable Outcomes among TB Patients in Two States in South India. Biomedical Central Public Health, 20, Article No. 1158. https://doi.org/10.1186/s12889-020-09257-5

[11] Ivers, L.C. and Walton, D.A. (2020) COVID-19: Global Health Equity in Pandemic Response. American Journal of Tropical Medicine and Hygiene, 102, 1149-1150. https://doi.org/10.4269/ajtmh.20-0260 https://www.ncbi.nlm.nih.gov/pmc/articles/PMC7253101/

[12] Cambanis, A., Yassin, M.A., Ramsay, A., Squire, S.B., Arbide, I., Cuevas, L.E., et al. (2005) Rural Poverty and Delayed Presentation to Tuberculosis Services in Ethiopia. Tropical Medicine and International Health, 10, 330-335. https://doi.org/10.1111/j.1365-3156.2005.01393.x

[13] Mesfin, M.M., Newell, J.N., Walley, J.D., Gessessew, A. and Madeley, R.J. (2009) Delayed Consultation among Pulmonary Tuberculosis Patients: A Cross Sectional Study of 10 DOTS Districts of Ethiopia. Biomedical Central Public Health, 9, Article No. 53. https://doi.org/10.1186/1471-2458-9-53

[14] Alobu, I., Oshi, S.N., Oshi, D.C. and Ukwaja, K.N. (2014) Risk Factors of Treatment Default and Death among Tuberculosis Patients in a Resource-Limited Setting. Asian Pacific Journal of Tropical Medicine, 7, 977-984. https://doi.org/10.1016/S1995-7645(14)60172-3 https://www.sciencedirect.com/science/article/pii/S1995764514601723

[15] Nagu, T.J., Spiegelman, D., Hertzmark, E., Aboud, S., Makani, J., Matee, M.I., et al. (2014) Anemia at the Initiation of Tuberculosis Therapy Is Associated with Delayed Sputum Conversion among Pulmonary Tuberculosis Patients in Dar-es-Salaam, Tanzania. PLoS ONE, 9, Article ID: e91229. https://doi.org/10.1371/journal.pone.0091229

[16] Sulis, G., Roggi, A., Matteelli, A. and Raviglione, M.C. (2014) Tuberculosis: Epidemiology and Control. Mediterranean Journal of Hematology and Infectious Diseases, 6, Article ID: e2014070. https://doi.org/10.4084/mjhid.2014.070

[17] Waitt, C.J. and Squire, S.B. (2011) A Systematic Review of Risk Factors for Death in Adults during and after Tuberculosis Treatment. The International Journal of $\mathrm{Tu}$ berculosis and Lung Disease, 15, 871-885. https://doi.org/10.5588/ijtld.10.0352 